\title{
Initiation of Coronal Mass Ejections
}

\author{
Ronald L. Moore and Alphonse C. Sterling \\ NASA Marshall Space Flight Center, XD12/Space Science Branch, Huntsville, Alabama
}

This paper is a synopsis of the initiation of the strongfield magnetic explosions that produce large, fast coronal mass ejections. The presentation outlines our current view of the eruption onset, based on results from our own observational work and from the observational and modeling work of others. From these results and from physical reasoing, we and others have inferred the basic processes that trigger and drive the explosion. We describe and illustrate these processes using cartoons. The magnetic field that explodes is a sheared-core bipole that may or may not be embedded in surrounding strong magnetic field, and may or may not contain a flux rope before it starts to eplode. We describe three different mechanisms that singly or in combination can trigger the explosion: (1) runaway internal tether-cutting reconnection, (2) runaway external tethercutting reconnection, and (3) ideal MHD instability or loss or equilibrium. For most eruptions, high-resolution, high-cadence magnetograms and chromospheric and coronal movies (such as from TRACE and/or SolafB) of the pre-eruption region and of the onset of the eruption and flare are needed to tell which one or which combination of these mechanisms is the trigger. Whatever the trigger, it leads to the production of an erupting flux rope. Using a simple model flux rope, we demonstrate that the explosion can be driven by the magnetic pressure of the expanding flux rope, provided the shape of the expansion is "fat" enough.

\section{INTRODUCTION}

An intense, days-long solar energetic particle (SEP) storm in interplanetary space, one that could kill astronauts and spacecraft, is generated by the bow shock of a large, fast coronal mass ejection (CME) (Kahler 1992; Reames 1999, 2001). The driving $\mathrm{CME}$ is a magnetic explosion that also produces a flare in the source region on the Sun (MacQueen \& Fisher 1983; Moore et al 2001; Falconer et al 2002). The magnetic fields that explode to drive the fastest CMEs and the most dangerous particle storms are in active regions with large sunspots, the regions of the strongest magnetic fields found on the Sun (e.g., Gopalswamy et al 2004).

Plate 1 shows a CME that is representative of those that drive SEP storms. A SOHO/LASCO difference image captures this $\mathrm{CME}$ as it blasts through the outer corona. Here, and in the original (non differenced) LASCO images, this CME shows the three-part bubble structure (bright envelope around a dark void with a bright core) typical of large, fast CMEs. Plate 1 also shows the onset of the magnetic explosion as observed by $\mathrm{SOHO} / \mathrm{EIT}$ in $\mathrm{Fe} \mathrm{XII}$ images. Although the magnetic field was tø weak to have sunspots, and hence was weaker than in many still stronger CME explosions, the explosion produced a large $\mathrm{CME}$ that was fast enough [900 $\mathrm{km} / \mathrm{s}$ (SOHO/LASCO CME Catalog)] to drive a bow shock and SEP storm. No SEP storm from this CME was observed at $\mathrm{SOHO}$ because $\mathrm{SOHO}$ was shielded from the particles by the interplanetary magnetic field: the CME occurred in a sector of the interplanetary magnetic spiral far behind of that of SOHO and Earth.

The magnetogram and Fe XII coronal images in Plate 1 show that the exploding field initially was a closed arcade with a dark filament of chromospherie temperature plasma suspended in its core. The filament stands above the neutral line (polarity dividing line) straddled by the arcade and shows that the magnetic field in the core of the arcade is strongly 


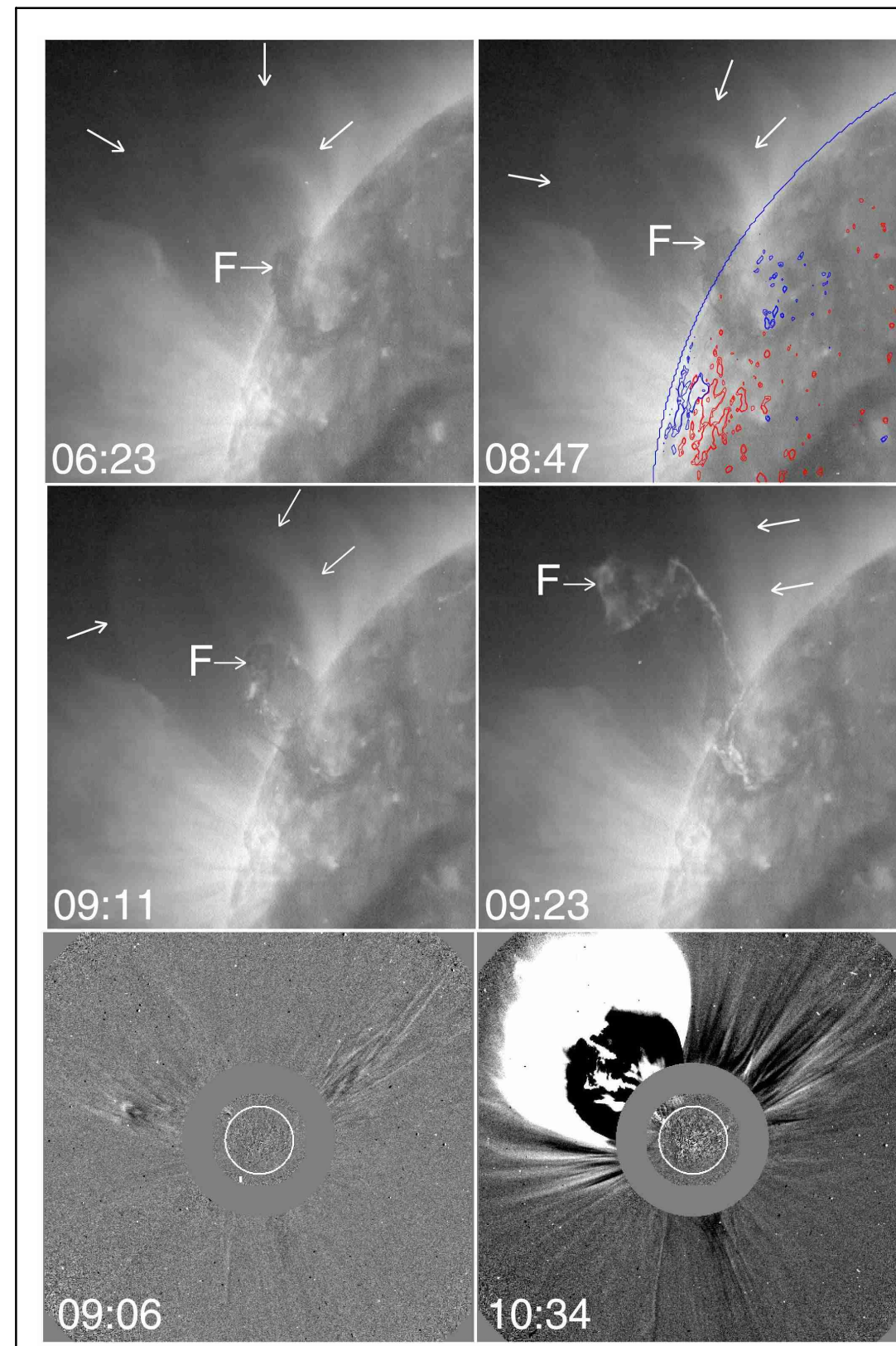

Plate 1. Onset of a typical filament-eruption magnetic explosion and the resulting large, fast CME. This eruption was observed by SOHO on 2002 January 4, and has been studied by Sterling and Moore (2004b). The universal time of each image is in the lower left corner. The uppe four panels are EIT $195 \AA$ Fe XII coronal images. The arrow labeled $\mathrm{F}$ points to the filament before it started erupt (first panel), during the slowrise onset of its eruption (second panel), and during the explosive fastrise phase (third and fourth paels). The other arrows point to the bright arcade that envelops the filament cavity and filament, and that $i$ blown open by their eruption. The superposed MDI magnetogram in the second panel shows the quadrupolar arrangement of positive plarity (red) and negative polarity (blue) magnetic flux in which the filament and cavity are seated. The bottom two panels are LASCO/C2 coronagraph runningdifference images of the outer corona at the start of the fastise phase of the filament eruption (left) and well after the CME had emerged from behind the aculting disk (right). The white circle outlines the solar disk catered behind the occulting disk. sheared: the direction of the field threading the filament is nearly parallel to the neutral line rather than nearly orthogonal as it would be if the field were in its minimumenergy potential configuration. That is, the magnetic location and form of the filament show that the core field is greatly deformed from its potential configuration and hence has a large store of nonpotential (free) magnetic energy that in principle is available for release in an explosion.

Over the past three decades, there has been a synthesis of various complementary observations of many ejective solar eruptions similar to that in Plate 1. These observations include chromospheric images, line-ofsight magnetograms, vector magnetograms, and coronal X-ray and EUV images of the eruption region, and chromospheric, coronal, and hard X-ray movies of the erupting filament-carrying sheared core field and the ensuing heated foot points and loops of the flare. From these observations a strong case has been made that (1) the pre-eruption configuration of the field that explodes to become a fast CME is typically a sheared core arcade like that in Figure 1, (2) there is enough free magnetic energy in the sheared core field to produce the explosion, and (3) the energy going into the $\mathrm{CME}$ and flare comes from the expanding sheared core field as it erupts in the explosion (Hirayama 1974; Kopp \& Pneuman 1976; Heyvaerts et al 1977; Moore \& LaBonte 1980; Moore et al 1980, 1987, 1995, 1997, 2001; Moore 1988, 2001; Shibata et al 1995; Shibata 1998; Canfield et al 1999; Sterling et al 2000, 2001a,b; Sterling \& Moore 2001a,b, 2003, 2004a,b).

The three-dimensional configuration of a sheared-core arcade prior to eruption is sketched in the first panel of Figure 1. In 


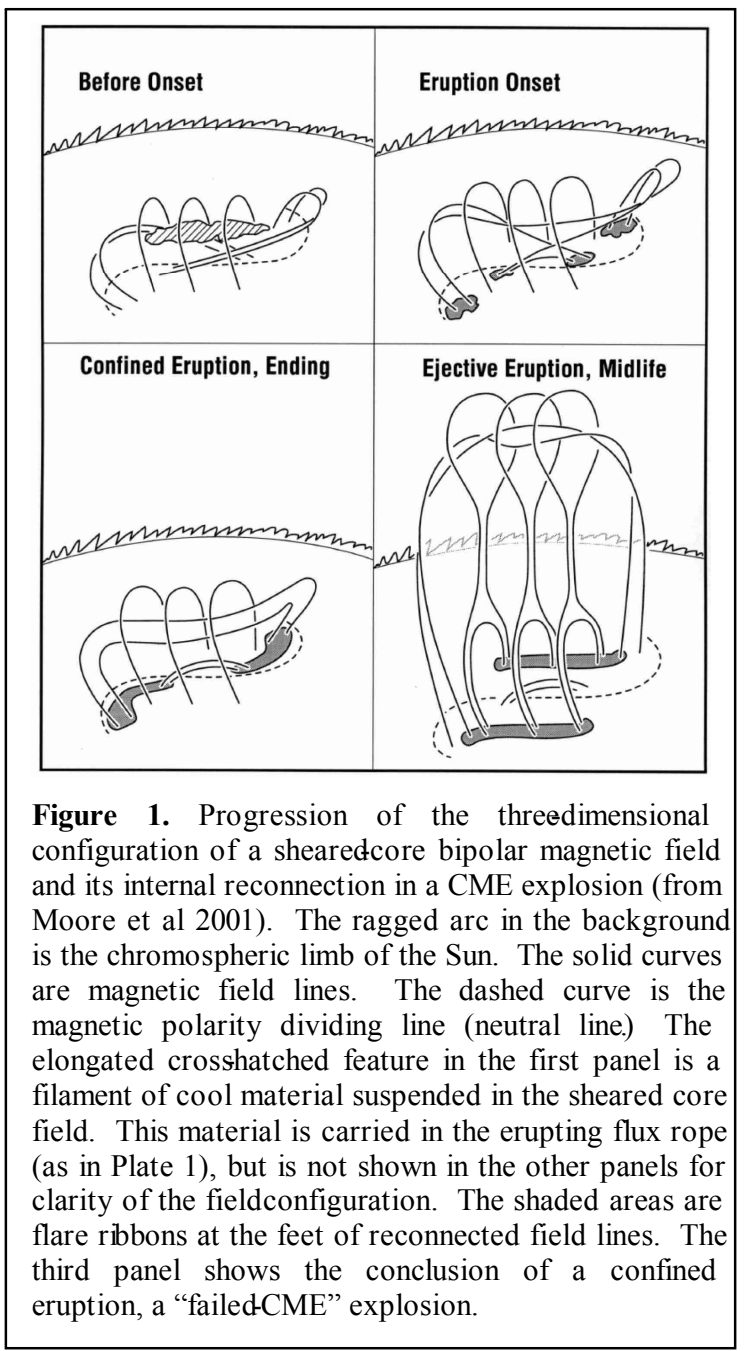

some cases, the sigmoidal shape of the sheared core field is as symmetric and as obvious in coronal X-ray images as it is in this drawing, but in many cases it is distorted, asymmetric, and much less obvious [e.g., see examples in Sterling et al (2000)]. This drawing shows only the closed bipolar field that has the sheared field and filament in its core. Any sheared core bipole on the Sun is embedded in other magnetic fields rooted around it more or less as in Plate 1. If the surrounding fields are of strength and span comparable to those of the shearedcore bipole, they can strongly influence whether and how the sheared core bipole explodes (Antiochos 1998; Antiochos et al 1999). If the surrounding fields are weak enough compared to the bipole, their effect on the initiation and growth of the explosion can reasonably be ignored. This is the case depicted in Figure 1.

At chromospheric and low coronal heights in sunspot active regions, the magnetic field strongly dominates the weight and pressure of the plasma
(Gary, 2001). As a result, the equilibrium configuration of the field is very nearly the forcefree configuration that the field would haveif the plasma had no weight and no pressure, the configuration determined by the balance between the outward push of magnetic pressure and the inward pull of magnetic tension (e.g., Cowling 1957). So, in an activeregion sheared-core bipole the cool filament material and the hot coronal plasma are constrained to trace the field lines, and do not appreciably deform the field from its force-free configuration. The elongation and striation of the filament show that the core field is strongly sheared, and the coronal plasma often illuminates the overall form of the shearedcore bipole, showing that it typically has the sigmoidal character of the initial sketch in Figure 1 (Canfield et al 1999; Sterling et al 2000).

In this paper, we consider the onset of CME explosions in which, until it begins to explode, the driving magnetic field is a nearly force free shearedcore bipole of the sigmoidal form sketched in Figure 1. The feet of all the field lines are locked to the massive body of the Sun. The manetic pressure pushes against the photosphere and outward in all directions, trying to explode the field, but is held in check by the magnetic tension pulling back toward the feet. So, for the case of forcefree equilibrium in the part of the preeruption field that is the source of the energy released in the explosion, the problem of the initiation of the CME explosion is that of how this equilibrium is destabilized or broken, so that the magnetic pressure is unleashed to drive the explosion.

We present three basic alternatives for triggering the explosion: internal tether cutting, external tether cutting (breakout), and ideal MHD instability or loss of equilibrium. The presentation outlines our current view of the eruption onset, based on our own observations and on the observations and theoretical work of others. In our view, for the strongield (initially force-free) case we are considering, the three presented alternatives cover the range of possibilities for triggering the explosion. Each alternative is illustrated by cartoons represerting the driving field, a surrounding field, and their interaction. No observations other than in Plate 1 and no numerical simulations of CME onsets are shown, but published observations and modeling studies are cited and briefly discussed in relation to each alternative. Our exposition of CME initiation is similar in approach to both that of Moore and Roumeliotis (1992) and that of Klimchuk (2001). It is an extension of Moore and Roumeliotis in that they did not consider the role of surrounding magnetic 
fields. Also in contrast to Moore and Roumeliotis, we do not explicitly portray the role of emerging magnetic fields in destabilizing the sheared-core bipole; in our scheme, reconnection between crossed strands of the sheared core field has basically the same role. Our considerations of CME initiation are narrower in scope than those of Klimchuk (2001), which included the possibilities of either plasma weight or plasma pressure being important in causing the explosion, and the possibility that the energy for the explosion comes from below the photosphere during the explosion. We limit our considerations to the force-free situation appropriate for the initiation of fast CMEs that come from strongfield regions and that are diven by the release of magnetic energy stored above the photosphere. Within this scope, our approach is a refinement of that of Klimchuk (2001). In Klimchuk (2001), the basic alternatives for CME initiation are illustrated by cartoons of analogous simple mechanical systems (springs, weights, strings, and pulleys) and thermal explosions (bombs). We illustrate our alternatives by cartoons that more directly represent the magnetic field in observed CME explosions in strong-field regions.

For each of the thee trigger mechanisms, we describe the initiation of the CME explosion with the aid of a sequence of three cartoons of the magnetic field (before eruption onset, just after eruption onset, and after the eruption is well underway) for the case in which the erupting sheared core field is in the central lobe of a quadrupolar field. It is observed that when a sheared core field (traced by a filament) erupts, the eruption often begins with a relatively long-lasting slow-rise phase (having little acceleration and during which the filament noticeably ascends) and then rather abruptly transitions to an explosive fast-rise phase of strong acceleration (e.g., Kahler et al1988; Sterling and Moore 2004a,b, 2005). In the quadrupolar cases that we depict in our cartoons, there are two places that reconnection can occur: (1) at the magnetic null (Xpoint) above the erupting central lobe, and (2) between the sheared legs of the erupting core field (below the erupting filament). Reconnection at the null is expected to produce coronal and chromospheric brightening in the two side lobes of the quadrupole, and reconnection below the filament is expected to produce coronal and chromospheric brightening in the sheared core field. The expected brightenings have been observed in many eruptions, and are taken as evidence of the reconnection (e.g., Aulanier et al 2000; Gary and Moore 2005; Sterling and Moore 2001a,b, 2004a,b, 2005). In the cartoons, we use the label "slow runaway reconnection" for reconnection that occurs during the slow-rise phase of the eruption, and we use the label "explosive reconnection" for reconnection that occurs during the explosive phase of the eruption. These terms are not intended to mean that there is necessarily different physics in the reconnection in the two phases, but only that, as observed brightenings strikingly indicate, the reconnection is much faster and stronger in the explosive phase than in the slow-rise phase of the eruption.

In each of our initiation alternatives, sooner or later, reconnection within the sheared core field creates a flux rope that erupts upward, carrying the filament (if present) within it. Observations indicate that the explosion is driven by the release of magnetic energy from the erupting flux rope via its expansion (Moore 1988). We point out that in order for this to work, that is, in order for there to be a net decrease in the magnetic energy in the exploding flux rope, the shape of the expansion must be sufficiently "fat."

\section{CANNONICAL PRE-ERUPTION FIELD CONFIGURATION}

In Plate 1, the preeruption filament and sheared core field run along the middle neutral line of a triplet of roughly parallel neutral lines. These three neutral lines divide the polarity domains of a quadrupolar arrangement of magnetic flux thatspans about a solar radius laterally from southeast to northwest. This magnetic setting of a shearedcore bipole is shown schematically in Plate 2. The style of the depiction is that used by Antiochos et al (1999) in displaying their simulation of CME initiation by breakout reconnection. Whereas their simulation was for a $\mathbf{z}$ D global quadrupolar field that straddled the equator and circled the Sun, our sketch is for an analogous 3 $\mathrm{D}$ quadrupolar field of the scale of that in Plate 1 or smaller. The 3-D form of the central shearectcore bipole is the same as that in Figure 1 (except that the sense of the magnetic shear and twist in the sigmoid is right-hand rather than left-hand).

The situation depicted in Plate 2 is the ideal symmetric one, much more symmeric than in the example observed case in Plate 1, but having the same topology. Nearly symmetric preeruption quadrupolar configurations of this topology do occur (e.g., Sterling \& Moore 2004a). In other observed quadrupolar situations, the shearedcore bipole is one of the side lobes rather than the central lobe (e.g., Sterling \& Moore 2004b). However, the situation in Plate 2 encompasses our three basic alternatives for CME initiation in basically the same way as for any multi-polar configuration in which there is an 


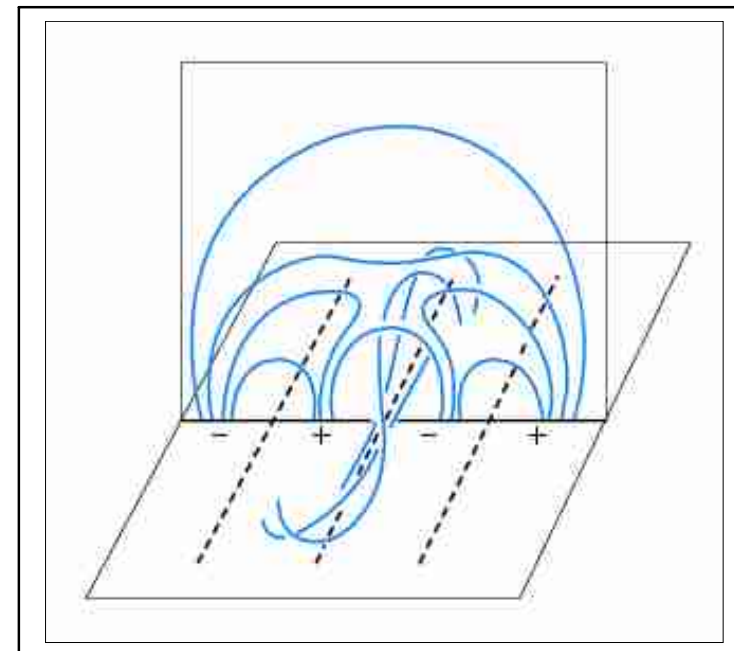

Plate 2. The three-dimensional topology of the quadrupolar magnetic fiel configuration for $\mathrm{CME}$ explosions such as in Plate 1. This is the canonical configuration, which has the exploding sheared core field in the central lobe of the quadrupole. As in Figure 1, the solid curves are field lines and the dashed lines are polarty dividing lines. The polarity of the field on each side of each of the three neutral lines is specified by a plus sign or a nimus sign, and matches the polarity arrangement in Plate 1.

embedded sheared-core bipole with an external magnetic null somewhere above it. In this canonical configuration, there are two places where reconnection can begin: within the sheared core field as depicted in Figure 1, or at the magnetic null between the envelope of the shearedcore central lobe and the oppositely-directed overarching envelope of the quadrupole. In the following section, for each of our CME-initiation alternatives, we depict the 3D field configuration of Plate 2 with $2 \mathrm{D}$ cartoons representing this configuration viewed horizontally along the direction of the neutral lines.

\section{ERUPTION ONSET}

In this section we describe how the three alternatives for CME initiation would occur in our canonical single-bipole and quadrupolar field configurations, and consider their observable signatures.

\subsection{Eruption Triggered by Internal Tether-Cutting Reconnection}

Our first alternative for CME initiation is depicted by the 2-D cartoons in Plate 3 . Here, before eruption onset, the 3-D sigmoidal sheared core field sketched in $3-\mathrm{D}$ in Figure 1 and Plate 2 is represented by the
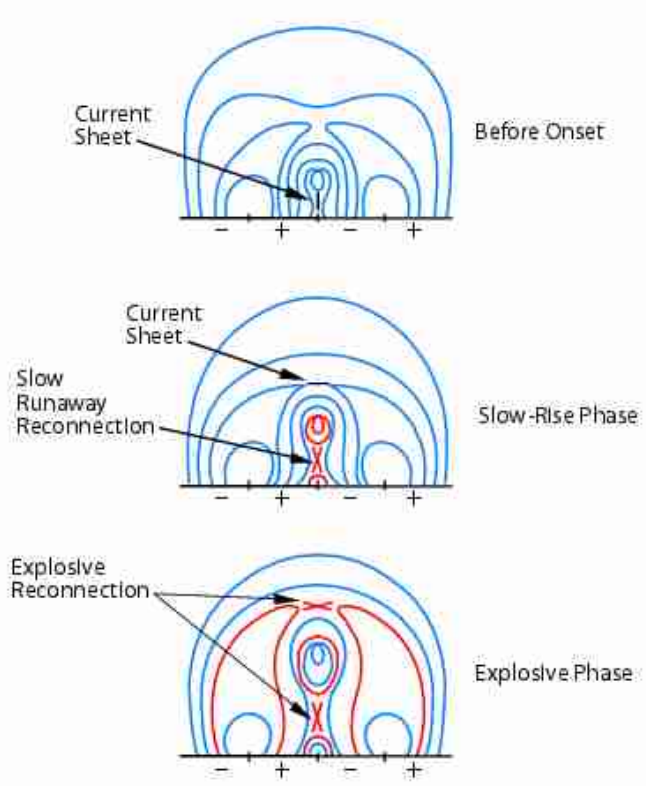

Plate 3. Onset of a quadrupolar CME explosion triggered by runawa internal tether-cutting reconnection. Here and in Plates 4 and 5, the drawings are 2-D renditions of the 3-D configuration in Plate 2 viewed from the front end.

innermost loop of the central lobe of the quadrupole. We have drawn this loop with a symbolic spiral dip in it to indicate that the core field is twisted and sheared along the neutral line and that it is thereby able to suspend cool filament material within it. Before eruption onset, the quadrupole is in overall force-free equilibrium. In particular, the magnetic pressure of the sheared core field is balanced by the combination of its own magnetic tension and the magnetic pressure and tension in the rest of the quadrupole. Also before eruption onset, the field around the magnetic null above the central lobe is sufficiently relaxed that there is no current sheetat the null. But we do have a current sheet low in the sheared core field, between the inner legs of the two elbows of the sigmoid where they shear past each other under the filament (as sketched in Figure 1). We suppose that this current sheet has beenformed by the legs being slowly pushed together by photospheric flows, perhaps involving flux cancellation at the neutral line as in the preeruption slow tether cutting of Moore and Roumeliotis (1992). The current sheet is the contact interface betweenthe two legs. Across this interface the vertical component of the sheared core field reverses direction. As it forms, the current sheet itself may or may not become nonforce-free before it is thin 
enough for reconnection to begin across it.

When the current sheet becomes sufficiently thin, reconnection begins as depicted in the second cartoons in Figure 1 and Plate 3. This is a runaway tether-cutting process as follows. The reconnected field lines above the reconnection site have had their number of footpoints cut from four to two (Figure 1), so that they are no longer tied to the photsphere under the filament and now run the length of the sigmoid. This releases them to erupt upward, which allows the inner legs of the sigmoid to further collapse together and drive more reconnection. The reconnection and eruption begin slowly, but due to this positive feedback the flux rope that is built and released by the reconnection becomes progressively farther out of force balance, and the eruption speed and reconnection rate progressively increase. In this manner, the CME explosion is initiated by the onset and runaway growth of tethercutting reconnection internal to the sheared core field (Moore and LaBonte 1980; Sturrock et al 1984; Sturrock 1989; Moore and Roumeliotis 1992).

As Figure 1 and Plate 3 indicate, this alternative for CME initiation is a possibility for either an isolated sheared-core bipole or a quadrupolar field having a sheared-core bipole within it. For a single-bipole CME explosion, the eruption of the unleashed flux rope is strong enough to overcome the restraint of the envelope of the bipole, the envelope field is blown out with the flux rope inside it as in Figure 1, and the legs of the "opened" envelope field reclose by reconnection in the wake of the CME expulsion. Early in the eruption, when the bipole is still closed, the reconnection produces a "four-ribbon" flare. As the reconnection seamlessly progresses from being between the closed sheared magnetic loops of the sigmoid early in the eruption to being between the stretched legs of the extruded envelope field late in the eruption, the flare loops that are formed and heated by the reconnection and issue downward from the rising reconnection site progress from being low and strongly sheared across the neutral line to becoming an increasingly less-sheared, growing arcade rooted in two separating flare ribbons (Figure 1). Such progression from four to two flare ribbons has been observed in ejective filament-eruption flares (e.g., Moore d al 1995).

No matter how the eruption of the sheared core field is triggered, and whether or not the exploding bipole is embedded in strong surrounding field, flare brightening indicative of internal tether-cutting reconnection is usually observed to begn early in the eruption (e.g., Moore and LaBonte 1980; Kahler et al 1988; Moore et al 1984, 1995, 2001; Sterling and
Moore 2004a,b, 2005). Because this reconnection produces closed flare loops and begins while the exploding bipole is still closed, only part of the bipole's field is "opened" in producing the CME (as in Figure 1). This is compatible with the AlySturrock theorem: no stressed closed magnetostatic field has enough free energy to explode itself entirely open (Aly 1991; Sturrock 1991).

Is internal tether-cutting reconnection observed to be the trigger of single-bipole CME explosions? It appears to be in cases in which flare brightening (presumably from reconnection) is observed to begin low in the sheared core field in near synchrony with the onset of the slow rise of the filament/flux rope (e.g., Moore and LaBonte 1980; Moore et al 2001; Sterling and Moore 2005). However, because of finite time resolution and brightness noise thresholds, it remains uncertain whether (1) the internal reconnection begins at the start of the rising motion (which would be evidence for internal tethercutting as the trigger) or (2) the rising motion begins first. That is, observations of any single-bipole eruption have not yet ruled out the possibility that the shered core field first begins to erupt via an ideal MHD instability or loss of equilibrium, and that this soon drives the production of a current sheet and reconnection low in the erupting field (e.g. Rust and Kumar 1996; Rust and LaBonte 2005). This possibility is our third alternative for CME initiation. Many magnetostatic and magnetohydrodynamic modeling studies of single-bipole CME onsets based on prescribed evolution of the magnetic flux and/or magnetic shear have found results favoring an ideal MHD trigger (e.g., Isenberg et al 1993; Titov \& Demoulin 1999; Amari et al 2000; Linker and Mikic 1995; Chen and Shibata 2000; Roussev et al 2003; Gibson et al 2004). Triggering of eruption in a single-bipole configuration by spontaneous onset of tether-cutting reconnection in the sheared core field has not yet been demonstrated by MHD modeling (Antiochos 2005). Observations of filament-eruption flares with early brightening at sites of emerging magnetic flux near the neutral line suggest that such flux emergence may be required for internal tether cutting reconnection to be the trigger in practice (e.g., Heyvaerts et al 1976; Moore et al 1984; Sterling and Moore 2005). That is, if the configuration is initially MHD stable, local flux emergence may be required to produce the internal current sheet and start the runaway reconnection.

When the sheared-core bipole is embedded in a quadrupolar field as in Plate 3, once an explosion is triggered in this bipole, it erupts upward, compresses the null, and soon drives reconnection there. Before 
the explosion is triggered, the null may or may not be so relaxed as to have no current sheet as we have drawn it in Plate 3. Either way, for our present alternative for $\mathrm{CME}$ initiation (triggering by internal tether cutting), there is no reconnection at the null until it is further compressed by the eruption from below. The eruption from below is triggered and starts to grow in the same way as in the singlebipole case. Once reconnection at the null begins, it amounts to external tether cutting and is a runaway (explosive) process in the same way as the internal tether-cutting reconnection. Before reconnecting, the field lines of the envelope of the shearedcore bipole and the field lines of the overall envelope of the quadrupole act to tie down the shearedcore bipole. The null-point reconnection cuts these tethers and produces reconnected field lines that sling themselves out the way. Hence, the external reconnection further unleashes the exploding bipole, which makes the explosion stronger and drives the reconnection faster.

In the present scenario (triggering by internal tether-cutting), the external reconnection at the null, once it gets started, is the same as the breakout reconnection in the model of Antiochos et al (899) and in our second alternative for $\mathrm{CME}$ initiation, and could equal or exceed the internal reconnection in further unleashing and growing the explosion. The essential difference between the present scenario and the breakout scenario is in the cause and effect relation between the onset of breakout econnection and the onset of internal tethercutting reconnection. In the present scenario, internal tether cutting bgins first and leads to breakout reconnection, whereas the opposite occurs in the breakout scenario.

In each of our three alternatives for the canonical quadrupolar situation, before and during its eruption, the central lobe has the form of the erupting bipole in Figure 1. The external reconnection strips away some of the outer envelope of the erupting bipole. While the erupting bipole is thereby breaking through the envelope of the quadrupole, internal reconnection is growing the flux rope above it and the flare arcade below it. Once the erupting bipole has broken out of the quadrupole, it explodes on out to become a CME and its legs continue to reclose, further growing the arcade as in Figure 1.

As indicated in Plate 3, reconnection driven at the null produces hot plasma on the reconnected field lines, resulting in bright coronal loops in the side lobes and remote flare brightening at the feet of these loops. If runaway internal tether-cutting reconnection is the trigger of the expbsion, and not breakout reconnection, then flare heating in the sheared core field should begin together with the onset of the filament eruption and before the onset of the heating effects of the breakout reconnection. We know of no published example of an observed embedded-bipole CME explosion onset in which this is clearly the case. However the number of pubished well-observed events that have been studied in this respect is still small $(<\sim 10)$. While most of these show evidence for breakout reconnection starting early in the eruption, some also show brightening in the core field early in the eruption, and soleave open the possibility that either internal tether cutting or MHD instability is the trigger (e.g., Sterling and Moore 20004a,b). It is also possible that any two or all three of our CME-initiation alternatives play together so closely from the begiming in some events that the trigger should not be assigned to only one alternative. Since internal tethercutting reconnection begins early in the onsets of what appear to be single-bipole CME explosions (Moore et al 2001), it will be surprising if no embedded-bipole CME explosions are triggered in the same way, with no initial help from breakout reconnection.

\subsection{Eruption Triggered by External Tether-Cutting Reconnection (Breakout)}

Our second alternative for CME initiation is depicted in Plate 4. This is similar to the first alternative in that the explosion of the sheared core field is again triggered by runaway tethercutting reconnection, but this time the econnection is at the external null rather than between the crossed legs of the core field. Unlike the other two alternatives, this alternative is not an option for the initiation of single bipole CME explosions because these, by definition, have no appreciably strong surrounding fields and hence can have no significant external tether cutting. When the exploding bipole is embedded in an arrangement of strong field giving an external null, as in our canonical quadrupolar configuration, the explosion can be triggered by reconnection at the null as follows.

It is supposed that photosphericallydriven evolution of the sheared core field, via shearing flows or further emergence of sheared core field, gradually inflates the middle lobe of the quadrupole without producing a current sheet within the sheared core field. This gradually compresses the feld at the null and produces a current sheet there that becomes progressively thinner. Eventually reconnection begins at the current sheet. This reconnection may begin slowly, but because it renders the shearedcore bipole progressively farther out of foce balance, it is a runaway process. As the filament/flux rope rises 


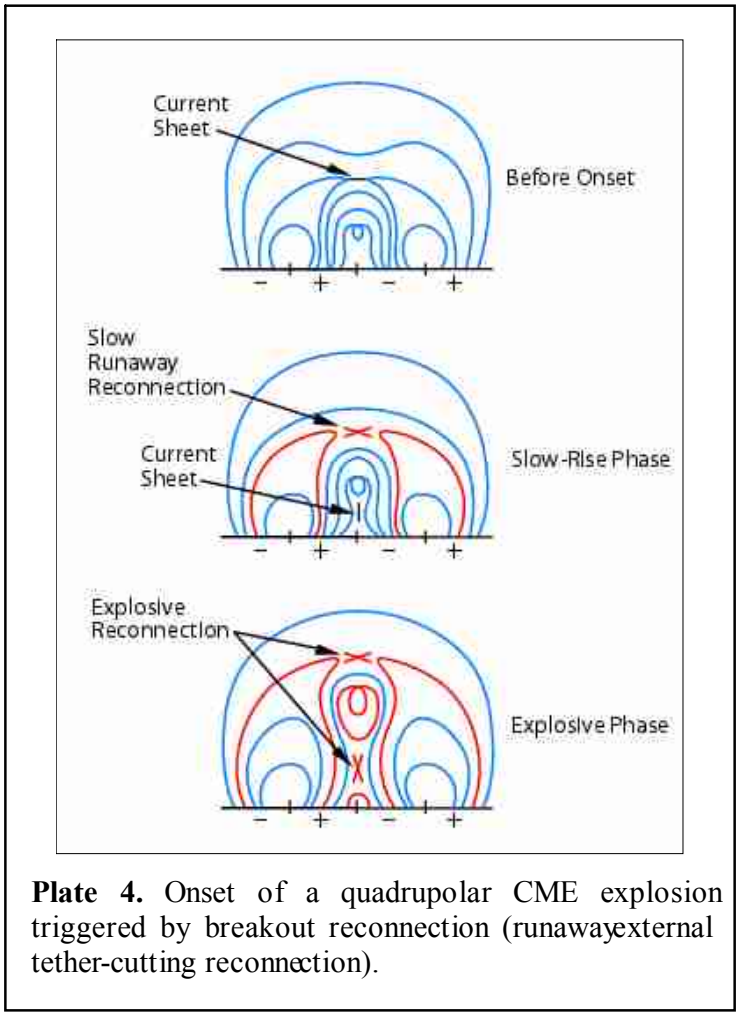

up, the stretched legs of the centrallobe core and envelope field begin to collapse together under it and form a current sheet at their interface (Plate 4, second cartoon). As the exernal reconnection and the core field eruption continue to grow, the internal current sheet grows and thins and runaway tethercutting reconnection is soon driven there as well, which further unleashes the explosion.

The sequence of events described above is that demonstrated by Antiochos et al (1999) in their numerical MHD 2-D simulation of the breakout scenario for CME initiation conceived by Antiochos (1998). In a CME explosion from a shearectcore bipole embedded in strong surrounding field with an opposing polarity arrangement, there will be a null more or less above the exploding bipole, and, regardless of how the explosion is triggered, the explosion will drive breakout reconnection at the null. Clear evidence for this reconnection has been observed in several such explosions (Aulanier et al 2000; Sterling et al 2001a,b; Sterling and Moore 2001a,b, 2004a,b; Gary and Moore 2004; Li et al 2005). Among these, the strongest cases for the explosion being triggered by breakout reconnection, that is, the cases having the most compelling evidence that remote flare brightening occurred before the onset of the filament/fluxrope eruption, are the event studied by Aulanier et al (2000) and the

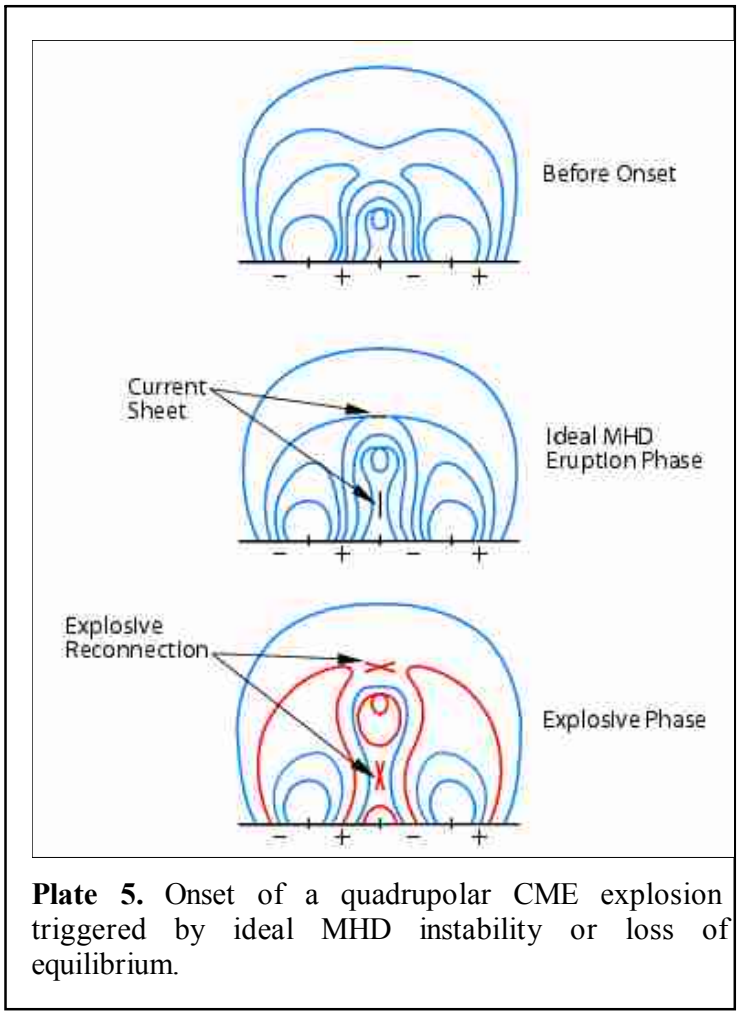

event studied by Gary and Moore (2004) and by (Li et al (2005). Even in these two cases, the observations do not rule out that the breakout reconnection started in response to an unnoticed ideal MHD convulsion in the sheared-core bipole. In the other cases, either no clear tracer of the onset of the core-field eruption (such as a slowly rising filament) is present or to within the time reslution of the observations the remote brightening begins together with the slow-rise phase of the filament eruption. So, in these cases the observations are consistent with either that the breakout reconnection triggered the slow-rise phase of the core field explosion or that the slow-rise onset was triggered by one or both of the other alternatives and initially drove the breakout reconnection.

\subsection{Eruption Triggered by Ideal MHD Instability or Loss of Equilibrium}

Our third alternative for CME initiation is shown in Plate 5. This alternative differs from the other two in that the first step in the eruption, the trigger, does not involve reconnection. Like the internal reconnection alternative, it is a possibility for the trigger whether or not the sheared-core bipole is embedded in strong surrounding field. In the quadrupolar case in Plate 5, before eruption onset there is no current sheet either 
at the null or in the sheared legsof the core field, or if there is, neither current sheet is yet thin enough for reconnection to start. It is supposed that photospherically-driven evolution of the flux distribution, perhaps including flux emergence and/or cancellation, gradually evolves the field configuration in the central lobe until the field in the filament/flux rope is so twisted and/or untethered that its force-free magnetostatic equilibrium becomes unstable (e.g., to kinking) or untenable (the core field looses its equilibrium and seeks a new equilibrium by erupting upward). Magnetostatic and magnentohydrodyamic 2-D and 3-D modeling studies of this scenario have shown (at least in singlebipole situations) that such dynamic behavior can be initiated without resistive dissipation (reconnection) at current sheets (e.g., Isenberg et al 1993; Linker and Mikic 1995; Titov and Demoulin 1999; Amari et al 2000; Chen and Shibata 2000; Roussev et al 2003; Gibson et al 2004). In these bipolar models, the instability or loss of equilibrium leads to the formation of a current sheet below or low in the erupting flux rope, between the legs of the envelope field or in the fold of a kink in the flux rope. In the quadrupolar situation in Plate 5, the upward eruption in the central lobe also produces a current sheet at the null. As soon as either current sheet is thin enough, runaway reconnection occurs there. As in the other two alternatives, this tether cutting further unleashes the explosion of the central lobe and helps the eruption open the quadrupole and become a CME (Plate 5, third cartoon). (Again, we emphasize the difference between an ideal MHD trigger and either of the two tethercutting options for the trigger: only in the ideal MHD option does the eruption begin without any pre-existing current sheets, or if any current sheets are present, without any reconnection at these current sheets. Ideal MHD instability or loss of equilibrium starts the eruptive motion, even though, as the above modeling studies indicate, this soon produces one or more reconnection current sheets and the subsequent reconnection is essential for the production of a fultblown CME.)

The amount of twist displayed by sigmoidal core fields before or during eruption onset suggests that the eruption may be triggered bykink instability, and many erupting filament/flux ropes doe appear to kink as they erupt (Rust and Kumar 1996; Rust and LaBonte 2005). While these observations are consistent with triggering by ideal MHD instability, in observed eruption onsets this altemative is difficult to distinguish from the other two, unless the formation of the tethercutting current sheets is sufficiently delayed from the start of the ideal MHD eruption. As noted in the Introduction, observed filament/flux rope eruptions often begin with a slowrise phase that persists with little acceleration until the filament has ascended markedly and then rapidly transitions to an explosive fastrise phase of strong acceleration (Sterling and Moore 2004a,b, 2005). During the slow-rise phase, if there is internal tethercutting reconnection or external breakout reconnection in progress, it is apparently slow and its heating effects (flare brightening) should be relatively weak [as is seen in Moore et al (2001) and in Sterling and Moore (2004a)] or perhaps below detection threshold (Sterling and Moore 2003). In the few cases we have studied so far, the brightening becomes obvious before or soon after the start of the fast phase of the eruption. As a rule, the stronger the erupting magnetic field, the faster the eruption develops, and the stronger the flare heating. If reconnection heating is occurring in the slowrise phase, it should be easier to detect in activeregion eruptions than in quiet-region eruptions, given adequate time resolution. So, if an activeregion filament eruption were observed to enter its fast phase with no sign of flare heating anywhere in the active region, that would be strong evidence for initiation by an ideal MHD process. To our knowledge, no such strongfield filament eruption has yet been observed in either a bipolar or multi polar setting. If MHD-triggered eruptions do occur, they will be easier to detect in bipolar situations than in multi-polar situations if, as seems likely, the MHD eruption takes longer to produce a reconnecting current sheet in the legs of the erupting bipole than at an external null.

\section{SHAPE OF EXPANSION OF THE ERUPTING FLUX ROPE}

In CME explosions of sigmoidal sheared core fields, whether the pre-eruption sigmoid is in an isolated bipole as in Figure 1 or in an embedded bipole as in Plate 2, and regardless of which of our three alternatives or any combination of these triggers the eruption, observations show that by the time the filament-carrying field has erupted to a height of order the original length of the sigmoid, internal tether-cutting reconnection is in progress between the legs of the erupting bipole (e.g., Sterling and Moore 2004b, 2005). For example, the last Fe XII image in Plate 1 captures the erupting filament at about this height and shows flare ribbons brightening along the neutral line. Before the eruption, it is reasonable to consider the field lines holding the filament to comprise a flux rope, but these field lines may or may 
not run the length of the sigmoid withoutrooting in the photosphere. By the time internal reconnection is well underway and producing flare ribbons as in Plate 1, any ties of the filament field to the photosphere under it have apparently been cut, and the filament can be considered to be carried in an erupting flux rope then and after, if not before. It appears to be the expansion of the field in this flux rope that drives the explosion, depleting the magnetic energy in the flux rope (Moore 1988).

For the magnetic energy content of the flux rop to decrease as it erupts, it must expand in cross sectional area enough faster than it expands in length. That is, the shape of the expansion must be sufficiently "fat." We will demonstrate this requirement by using the simple cylindrical model flux rope shown in Figure 2. For simplicity, and because sigmoids and filaments in active regions are only mildly twisted, having no more than a full turn from end to end, we ignore the twist. We also ignore the curvature of the erupting rope and the plasma pressure in the rope, and take the magnetic field to be uniform inside the model rope and the cross section to be constant along the length. At any instant in the eruption, the magnetic energy content $F_{\text {nag }}$ of the model flux rope is the magnetic energy density times the volume:

$$
\mathrm{F}_{\text {mag }}=\left(\mathrm{B}^{2} / 8 \pi\right) \mathrm{A} l=\left(\Phi^{2} / 8 \pi\right) l / \mathrm{A},
$$

where $\mathrm{B}$ is the field strength, A and $l$ are the cross sectional area and length of the rope, and $\Phi$ is the magnetic flux in the rope $(\Phi=\mathrm{BA})$. Due to he high electrical conductivity, the magnetic field in the rope obeys the frozen-in condition and defines the flux rope, and $\Phi$ remains constant as the rope expands. So, differentiation of Equation (1) gives

$$
\left(\Delta \mathrm{E}_{\mathrm{mag}}\right) / \mathrm{E}_{\mathrm{mag}}=(\Delta l) / l-(\Delta \mathrm{A}) / \mathrm{A}
$$

for the incremental change in the magnetic energy content from incremental changes in the length and area of the rope as it expands. For the expansion of the flux rope to be driving the explosion, the magnetic energy content of the rope must be decreasing $\left(\Delta \mathrm{E}_{\mathrm{mag}}<0\right)$, which requires

$$
\Delta \mathrm{A}) / \mathrm{A}>(\Delta l) / l \text {. }
$$

Thus, the shape of the expansion, $[(\Delta \mathrm{A}) / \mathrm{A}] /[(\Delta l) / l]$, must be "fatter" than unity for the explosion to proceed. If the area expands at the same fractioal rate as the length or slower, the magnetic energy content of the rope remains constant or increases, and

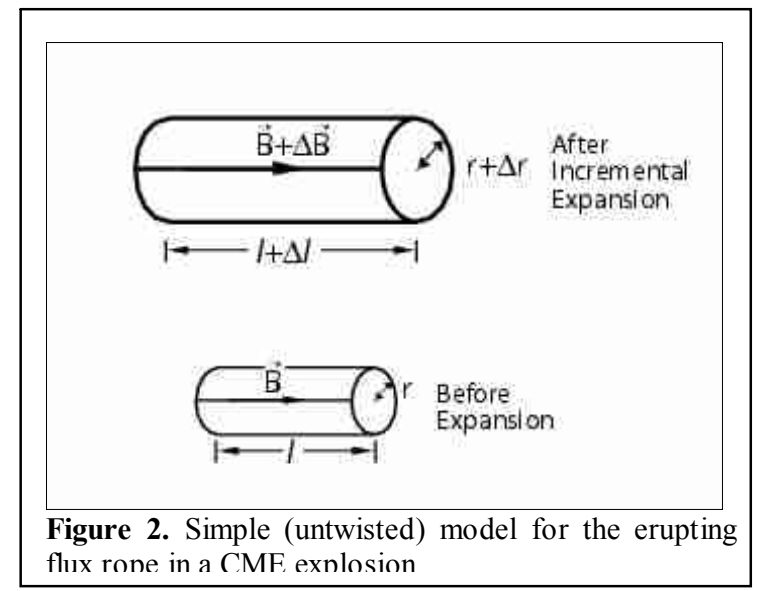

the explosion has to be driven by something other than the expansion of the flux rope. Twist in the flux-rope field would lower the above limit on the expansion fatness, because the energy in the component of the field perpendicular to the length of the rope would be decreased by expansion of the rope along its length.

In Plate 1, the expansion of the erupting flux rope (the erupting filament and filament cavity) appears to be roughly isotropic; that is, the diameter of the filament cavity and the length of the filament appear to increase at roughly the same rate. For isotropic expansion, $\mathrm{r} \propto l$, where $\mathrm{r}$ is the radius of the flux rope, $\mathrm{A} \propto l^{2}$, and $\mathrm{E}_{\mathrm{mag}} \propto 1 / l$. So, in the CME explosion in Plate 1 , the magnetic energy of the fluxrope rapidly decreases as it erupts, consistent with the eplosion being driven by the magnetic pressure of the flux rope.

\section{SUMMARY AND DISCUSSION}

From observations of CME explosions from strong sigmoidal sheared core fields traced by filaments, it is nearly certain that the explosion is driven by the magnetic pressure in the erupting filamentcarrying flux rope that is unleashed as the explosion is triggered and grows. Befre it explodes, the sheared core field is in forcefree equilibrium; its magnetic pressure is balanced by its own magnetic tension together with the tension and pressure of surrounding fields. Gradual evolution of the arrangement and amount of magnetic flux in and around the sheared core field can eventually upset this equilibrium and trigger the explosion. When the sheared field is the core of an isolated bipole, there are two different possibilities for the triggering process: (1) runaway tether-cutting reconnection could begin inside the core field, or (2) the equilibrium could become MHD unstable or impossible without an abrupt change in 
the field configuration (loss of equilibrium). When the sheared-core bipole is embedded in surrounding strong fields arranged in polarity so that there is a magnetic null above the embedded bipole, the explosion could be triggered by either of the above two alternatives for single bipoles or by a third alternative: breakout reconnection, which is runaway tether-cutting reconnection at the external null.

So far as we know, good evidence for breakout reconnection being the trigger has been found in only a couple of observed quadrupolar eruptions. In many observed eruptions in multi-polar configurations, while it is clear that external tethercutting reconnection occurs, the observations permit the explosion to be triggered by one or both of the other two alternatives. Likewise, in observed singlebipole eruptions, there is often clear evidence for internal tether cutting reconnection starting early in the eruption, but in no case yet have the observations ruled out that the erption was initiated by ideal MHD instability or loss of equilibrium. There is no a priori reason that pairs or all three of our alternative mechanisms could not act in concert as the trigger in some situations. For most eruptions, sorting out from observations which of these various possibilities is the trigger apparently requires (at least) high cadence, high-resolution movies in chromospheric, transition-region, and coronal emission, such as are provided by TRACE and are expected from SolarB, along with high-cadence, high-resolution magnetograms.

However the eruption is triggered, the erupting sheared core field is or soon becomes a flux rope that carries the erupting filament within it and continues to be built and further unleashed by tethercutting reconnection below it. The expanding flux rope drives the explosion provided that its expansion results in a decrease in its magnetic energy contat. This occurs if the shape of the expansion is sufficiently "fat." Specifically, the rate of logarithmic increase in the cross-sectional area of the flux rope $[(1 / \mathrm{A}) \mathrm{dA} / \mathrm{dt}]$ must be enough faster than the rate of logarithmic increase in the length of theflux rope $[(1 / l) \mathrm{d} l / \mathrm{dt}]$. In observed active-region filament eruptions, the expansion often appears to be roughly isotropic [e.g., see the filament eruptions shown in Moore (1987), Kahler et al (1988), or Sterling and Moore (2004b, 2005)], which indicates that the magnetic energy of the erupting flux rope rapidly goes into the explosion. If an erupting flux rope were observed to have an expansion shape of only about unity or less $([(1 / \mathrm{A}) \mathrm{dA} / \mathrm{dt}] /[(1 l) \mathrm{d} l / \mathrm{dt}]<\sim 1)$, this would indicate that the eruption of the flux rope was not driven by the flux rope acting on its surroundings but by the surroundings acting on the flux rope.

Acknowledgments. This work was supported by NASA's Science Mission Directorate through the Solar and Heliospheric Physics Program and he Sun-Solar System Connection Guest Investigator Program of its EartiSun System Division. We acknowledge the use of the CME catalogue generated and maintained by the Center for Solar Physics and Space Weather of the Catholic University of America in cooperation with the Naval Research Laboratory and NASA.

\section{REFERENCES}

Amari, T., J. F. Luciani, Z. Mikic, and J. Linker, A twisted flux rope model for coronal mass ejections and twøibbon flares, Astrophys. J., 529, L49-L52, 2000.

Antiochos, S. K., The magneic topology of solar eruptions, Astrophys. J., 502, L181-L184, 1998.

Antiochos, S. K., C. R. DeVore, and J. A. Klimchuk, A model for solar coronal mass ejections,Astrophys. J., 510, 485493, 1999

Aulanier, G., E. E. DeLuca, S. K. Antiochos, R. A. McMulhe, and L. Golub, The topology and evolution of the Bastille Day flare, Astrophys. J., 540, 1126-1142, 2000.

Canfield, R. C., H. S. Hudson, and D. E. McKenzie, Sigmoidal morphology and eruptive solar activityGeophys. Res. Lett., 26, 627-630, 1999.

Chen, P. F., and K. Shibata, An emerging flux trigger mechanism for coronal mass ejections, Astrophys. J., 545, 524-531, 2000.

Cowling, T. G., Magnetohydrodynamics, Interscience, New York, 1957.

Falconer, D. A., R. L. Moore, and G. A. Gary, Correlation of the coronal mass ejection productivity of solar active regions with measures of their global nonpotentiality from vector magnetograms: Baseline results, Astrophys. J., 569, 1016-1025, 2002.

Gary, G. A., Plasma beta above a solar active region: rethinking the paradigm, Sol. Phys., 203, 71-86, 2001.

Gary, G. A., and R. L. Moore, Eruption of a multipteurn helical flux tube in a large flare: Evidence for external and internal reconnection that fits the breakout model of solar magnetic eruptions, Astrophys. J., 611, 545-556, 2004.

Gibson, S. E., Y. Fan, C. Mandrini, G. Fisher, and P. Demoulin, Observational consequences of a magnetic flux rope emerging into the corona,Astrophys. J., 617, 600-613, 2004.

Gopalswamy, N., S. Yashiro, A. Vourlidas, A. Lara, G. Stenborg, M L. Kaiser, and R. A. Howard, Coronal mass ejections when the Sun went wild, Bull. Am Astron. Soc., 36, 738, 2004.

Heyvaerts, J., E. R. Priest, and D. M. Rust, An emerging flux model for the solar flare phenomenon,Astrophys. J., 216, 123-137, 1977.

Hirayama, T., Theoretical model of flares and prominences. I: Evaporating flare model,Sol. Phys., 34, 323-338, 1974.

Isenberg, P. A., T. G. Forbes, and P. Demoulin, Catastrophic 
evolution of a forcefree flux rope: A model for eruptive flares, Astrophys. J., 417, 368-386, 1993.

Kahler, S. L., R. L. Moore, S. R. Kane, and H. Zirin, Filament eruptions and the impulsive phase of solar flaresAstrophys. $J ., 328,824-829,1988$.

Klimchuk, J. A., Theory of coronal mass ejections, inspace Weather, edited by P. Song,H. J. Singer, and G. L. Siscoe, pp. 143-157, 2001.

Kopp. R. A., and G. W. Pneuman, Magnetic reconnection in the corona and the loop prominence phenomena,Sol. Phys., 50, 85-98, 1976.

Li, J., D. L. Mickey, and B. J. LaBonte, The X3 flare of 2002 July 15, Astrophys. J, 620, 1092-1100, 2005.

Linker, J. A., and Z. Mikic, Disruption of a helmet streamer by photospheric shear,Astrophys. J., 438, L45-L48, 1995.

MacQueen, R. M., and R. R. Fisher, The kinematics of solar inner coronal transients, Sol. Phys., 89, 89-102, 1983.

Moore, R. et al, The thermal Xray plasma, in Solar Flares, edited by P. A. Sturrock, pp. 34409, Colorado Associated University Press, Boulder, 1980.

Moore, R., Solar prominence eruption, inEncyclopedia of Astronomy and Astrophysics, edited by P. Murdin, pp. 2691 -2695, Institute of Physics Publishing, Bristol, 2001.

Moore, R. L., Observed form and action of the magnetic field in flares, Sol. Phys., 113, 121-124, 1987.

Moore, R. L., Evidence that magnetic energy shedding in solar filament eruptions is the drive in accompanying flares and coronal mass ejections, Astrophys. J., 324, 1132-1137, 1988.

Moore, R. L., M. J. Hagyard, and J. M. Davis, Flare research with the NASA/MSFC vector magnetograph: Observed characteristics of sheared magnetic fidds that produce flares, Sol. Phys., 113, 347-352, 1987.

Moore, R. L., G. J. Hurford, H. P. Jones, and S. R. Kane, Magnetic changes observed in a solar flare,Astrophys. J., 276, 379-390, 1984.

Moore, R. L., and B. J. LaBonte, The filament eruption in the 3B flare of July 29, 1973: Onset and magnetic field configuration, in Solar and Interplanetary Dynamics, edited by M. Dryer and E. TandbergHanssen, pp. 207-210, Reidel, Dordrecht, 1980

Moore, R. L., T. N. LaRosa, and L. E. Orwig, The Wall of Reconnection-Driven Magnetohydrodynamic Turbulence in a Large Solar Flare,Astrophys. J., 438, 985-996, 1995.

Moore, R. L., and G. Roumeliotis, Triggering of eruptive flares: Destabilization of the preflare magnetic field configuration, in Eruptive Solar Flares, edited by Z. Svestka, B. V. Jackson, and M. E. Machado, pp. 698, Springer-Verlag, Berlin, 1992.

Moore, R. L., B. Schmieder, D. H. Hathaway, and T. D. Tarbell, 3-D magnetic field configuration late in a large two-ribbon flare, Sol. Phys,, 176, 153-169, 1997.

Moore, R. L., A. C. Sterling, H. S. Hudson, and J. R. Lemen, Onset of the magnetic explosion in solar flares and coronal mass ejections, Astrophys. J., 552, 848-883, 2001.

Reames, D. V., Particle acceleration on the Sun and in the heliosphere, Space Sci. Rev., 90, 413-491, 1999.

Reames, D. V., SEPs: Space weather hazard in interplanetary space, in Space Weather, edited by P. Song, H. J. Singer, and G. L. Siscoe, pp. 101107, AGU, Washington, D. C., 2001.

Roussev, I. I., T. G. Forbes, T. I. Gombosi, I. V. Sokv, D. L. De Zeeuw, and J. Birn, A threedimensional flux-rope model for coronal mass ejections based on a loss of equilibrium, Astrophys. J., 588, L45-L48, 2003.

Rust, D. M., and A. Kumar, Evidence for helically kinked magnetic flux ropes in solar eruptons, Astrophys. J., 464, L199-L202, 1996.

Rust, D. M., and B. J. LaBonte, Observational evidence of the kink instability in solar filament eruptions and sigmoids, Astrophys. J., 622, L69-L72, 2005.

Shibata, K., A unified model of solar flares, irObservational Plasma Astrophysics: Five years of Yohkoh and Beyond, edited by T. Watanabe, T. Kosugi, and A. C. Sterling, pp. 187-196, Kluwer, Dordrecht, 1998.

Shibata, K., S. Masuda, M. Shimojo, H. Hara, T. Yokayama, S. Tsuneta, T. Kosugi, and Y. Ogawara, Hoplasma ejections associated with compactloop solar flares, Astrophys. J., 451, L83-L85, 1995.

Sterling, A. C., H. S. Hudson, B. J. Thompson, and D. M. Zarro, Yohkoh SXT and SOHO EIT observations of "Sigmoid-to-arcade" evolution of structures associated with halo CMEs, Astrophys. J., 532, 628-647, 2000.

Sterling, A. C., and R. L. Moore, Internal and external reconnection in a series of homologous solar flares, $J$. Geophys. Res., 106, 25,227-25,238, 2001a.

Sterling, A. C., and R. L. Moore, EIT crinkles as evidere for the breakout model of solar eruptions,Astrophys. J., 560, $1045-1057,2001 b$.

Sterling, A. C., and R. L. Moore, Tethercutting energetics of a solar quietregion prominence eruption, Astrophys. J., 599, 1418-1425, 2003.

Sterling, A. C., and R. L. Moøe, Evidence for gradual external reconnection before explosive eruption of a solar filament, Astrophys. J., 602, 1024-1036, 2004a.

Sterling, A. C., and R. L. Moore, External and internal reconnection in two filament-carrying magnetic cavity solar eruptions, Astrophys. J., 613, 1221-1232, 2004b.

Sterling, A. C., and R. L. Moore, Slowrise and fast-rise phases of an erupting solar filament, and flare emission onset, Astrophys. J, 630, $1148-1159,2005$.

Sterling, A. C., R. L. Moore, J. Qiu, and H. Wang, It proxies for EIT crinkles: Further evidence for preflare "breakout" type activity in an ejective solar eruption,Astrophys. J., 561, 1116-1126, 2001a

Sterling, A. C., R. L. Moore, and B. J. Thompson, EIT and SXT observations of a quietregion filament eruption: First eruption, then reconnection, Astrophys. J., 566, L219-L222, $2001 b$.

Sturrock, P. A., The role of eruption in solar flare\$ol. Phys., 121, 387-397, 1989.

Sturrock, P. A., P. Kaufman, R. L. Moore, and D. F. Smith, Energy release in solar flares,Sol. Phys., 94, 341-357, 1984.

Titov, V. S., and P. Demoulin, Basic topology of twisted magnetic configurations in solar flares, Astron. \& Astrophys., 351, 707-720, 1999. 